\title{
Optimal design of a for middle-low-speed maglev trains
}

https://doi.org/10.1515/phys-2018-0024

Received November 9, 2017; accepted November 12, 2017

Abstract: A middle-low-speed maglev train is supported by an electromagnetic force between the suspension electromagnet (EM) and the steel rail and is driven by a linear induction motor. The capability of the suspension system has a direct bearing on safety and the technical and economic performance of the train. This paper focuses on the dependence of the electromagnetic force on the structural configuration of the EM with the purpose of improving performance of a conventional EM. Finally, a novel configuration is proposed of a hybrid suspension magnet, which combines permanent magnets and coils, in order to increase the suspension force while reducing the suspension power loss.

Keywords: Maglev train, EM, hybrid suspension

PACS: 41.20.-q, 02.60.Gf, 02.60.Pn

\section{Introduction}

As a new type of transportation technology, maglev trains have attracted attention due to their many advantages such as low noise, no pollution, good ability on slopes, small turning radius - making them suitable for urban transportation. The electromagnetic suspension (EMS) technology plays a critical role in the development of maglev trains, as they are suspended due to an attractive magnetic force produced by electromagnets. A hybrid suspension, mixing electromagnets with permanent magnets,

\footnotetext{
^Corresponding Author: Song Xiao: Key Laboratory of Magnetic Suspension Technology School of Electrical Engineering Southwest Jiaotong University, Chengdu, China, E-mail:

xiaosong@home.swjtu.edu.cn

Kunlun Zhang, Guoqing Liu, Yongzhi Jing: Key Laboratory of Magnetic Suspension Technology School of Electrical Engineering Southwest Jiaotong University, Chengdu, China

Jan K. Sykulski: Fellow, IEEE, Electronics and Computer Science, University of Southampton, Southampton SO17 1BJ, UK, E-mail: jks@soton.ac.uk
}

has been found to remarkably reduce power loss in the suspension system. Research reported in many countries - especially USA, Japan, Germany, South Korea and China - focused on the development of hybrid maglev trains with permanent-electro-magnetic suspension (PEMS) [16]. But until now this technology has not been commercialized. One of the critical issues to solve is the protection against mechanical contact when using permanent magnets to provide the force. In this paper, an expression for the non-linear magnetic force of a conventional electromagnet is examined and a novel structural configuration of a PEMS magnet proposed. Finally, the performance of an optimized PEMS magnet is compared with the conventional electromagnetic system.

\section{Structure of the suspension magnets}

Figure 1 shows a photograph of a middle-low-speed maglev train on the Changsha Airport Express line, designed mainly by the Southwest Jiaotong University, China. Each carriage of the maglev train has five suspension bogies, where four suspension magnets and one linear induction motor are installed on one side of each bogie. The structure of a single electromagnet unit is presented in Figure 2.

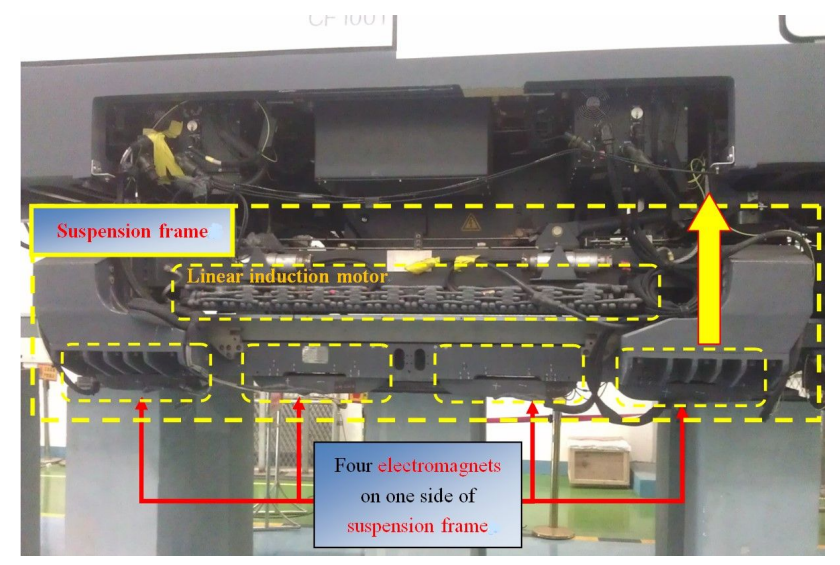

Figure 1: The electromagnets hanging on the suspension frame 


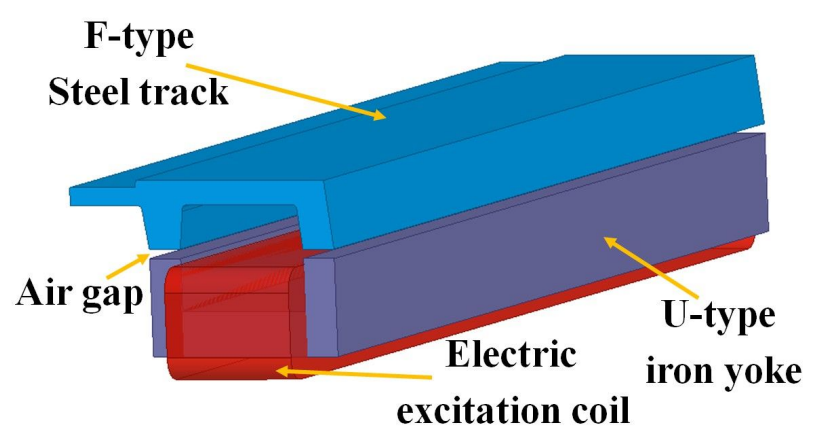

Figure 2: The configuration of the electromagnets

Further details of the structure of the electromagnets, as well as the F-type steel track, are shown in Figure 3. The symbols for the main system parameters are described in Table 2.

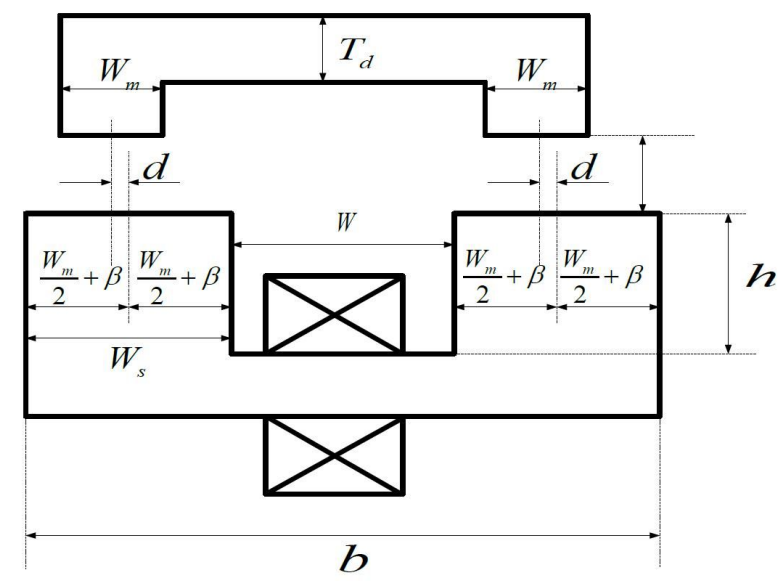

Figure 3: The diagram of the F-type track and the electromagnet

The magnetic field in the air gap can be assumed to be uniform if the air gap is small; the suspension force is then

$$
F_{0}=\frac{B^{2} W_{S}}{\mu_{0}} L
$$

where

$$
B=\frac{\mu_{0} \mu N I}{2 Z \mu+\mu_{0}\left[2 h+\left(1+W_{s} / T_{d}\right) b\right]} .
$$

A more accurate expression is provided in [4]

$$
\begin{aligned}
F_{Z}= & F_{0}\left[1+\frac{2 Z}{\pi W_{m}}+\frac{\beta-d}{\pi W_{m}} \operatorname{atan}\left(\frac{Z}{\beta-d}\right)\right. \\
& \left.+\frac{\beta+d}{\pi W_{m}} \operatorname{atan}\left(\frac{Z}{\beta+d}\right)\right]
\end{aligned}
$$

where $d$ is the value of the pole dislocation and $\beta$ is given by

$$
\beta=\frac{W_{s}-W_{m}}{2}
$$

Table 1: System Parameters of an Electromagnet

\begin{tabular}{ccc}
\hline System parameters & Symbol & $\begin{array}{c}\text { Value } \\
{[\mathrm{mm}]}\end{array}$ \\
\hline Magnetic pole width of steel rail & $W_{r}$ & 28 \\
Magnetic pole width of electromagnet & $W_{s}$ & 28 \\
Magnetic yoke height of suspension rail & $T_{d}$ & 21.5 \\
Magnetic yoke height of electromagnet & $h$ & 45 \\
Window width of suspension rail & $W$ & 112 \\
Air gap of suspension structure & $Z$ & 8 \\
Longitudinal length of suspension & $L$ & 2720 \\
structure & &
\end{tabular}

\section{The impact of the coil current and suspension gap on the electromagnetic force}

Using 2D Finite Element (FEM) simulations, the magnetic flux density and corresponding flux lines for both the Utype iron core and the F-type steel tracks under normal working conditions have been found as shown in Figure 4. The principal values used are: suspension gap $8 \mathrm{~mm}$; coil current $30 \mathrm{~A}$; the number of turns per coil 342 (other parameters as in Table 2). The suspension force produced by the EM system is $40.17 \mathrm{kN}$.

Figure 5 demonstrates the dependence of the suspension force on the coil current for three different air gaps. The force decreases when the size of the gap increases, with magnetic nonlinearity becoming pronounced for smaller gaps, as expected. Saturation effects are also clearly visible in the relationship of the force versus current, again in accordance with expectations.

The impact on the electromagnetic force of the lateral offset (with deviation $x$ ) caused by turning is shown in Figure 6. According to the publications, there are mainly two analytic methods to calculate suspension and guidance force. The first analytic method of calculating the suspension force $F_{z}$ and guidance force $F_{X}$ is defined in [7] as

$$
\left\{\begin{array}{l}
F_{z}=\frac{\mu_{0}}{4}\left(\frac{N i}{\delta}\right)^{2}\left(p w-x+\frac{2 x}{\pi}+\frac{2 x}{\pi} \tan ^{-1} \frac{\delta}{x}\right) L \\
F_{X}=-\frac{\mu_{0}(N i)^{2} L}{2 \pi \delta} \tan ^{-1} \frac{x}{\delta}
\end{array}\right.
$$

With the magnetic reluctance and leakage of yokes ignored.

Another expression, which also neglects the reluctance and leakage of yokes,may be found in [8]

$$
\left\{\begin{array}{l}
F_{z}=\frac{1}{4}(N i)^{2} \mu_{0}\left[\frac{(p w-x)}{\delta^{2}}+\frac{4 x}{4 \delta^{2}+\pi \delta x}\right] L \\
F_{X}=\frac{1}{4}(N i)^{2} \mu_{0}\left[-\frac{1}{\delta}+\frac{4}{4 \delta+\pi x}\right] L
\end{array}\right.
$$




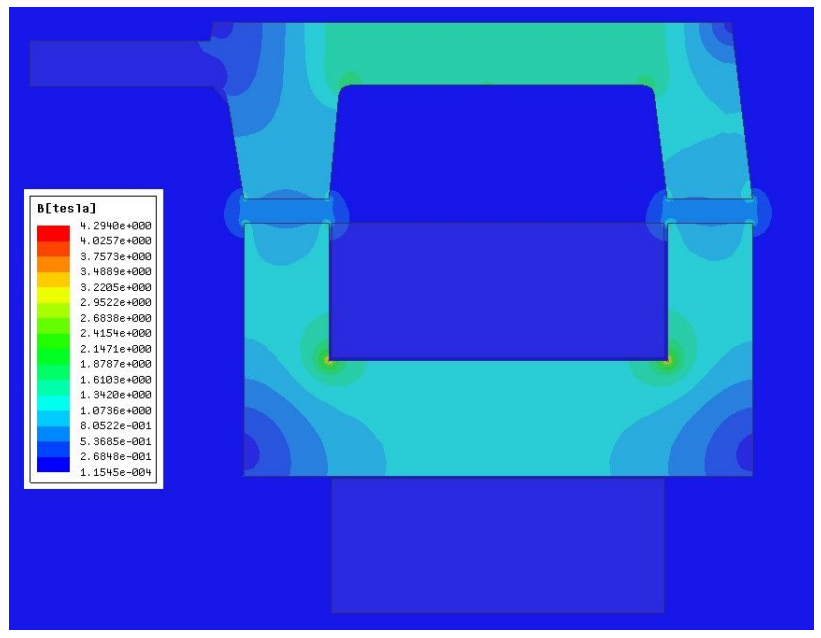

(a)

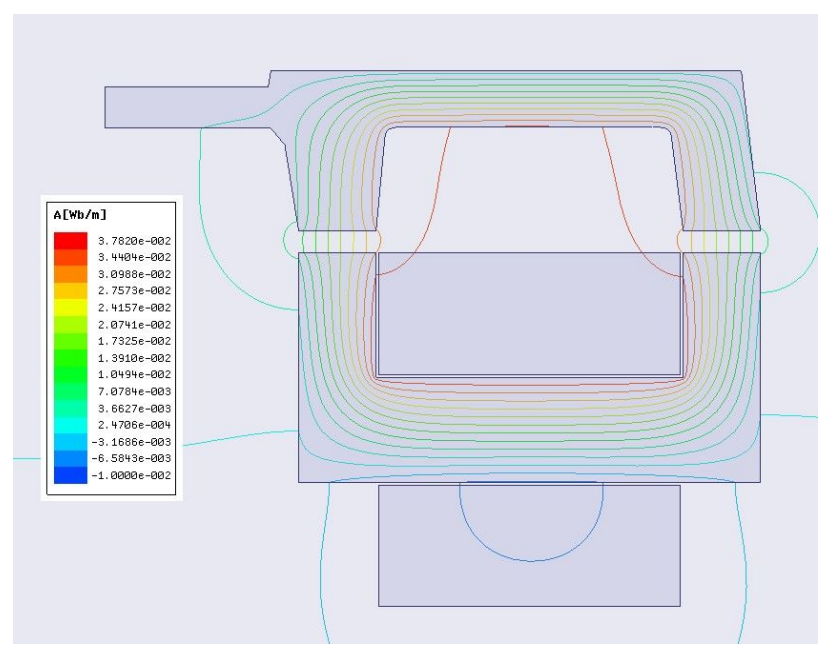

(b)

Figure 4: (a) Flux density distribution in the EM; (b) Corresponding flux lines

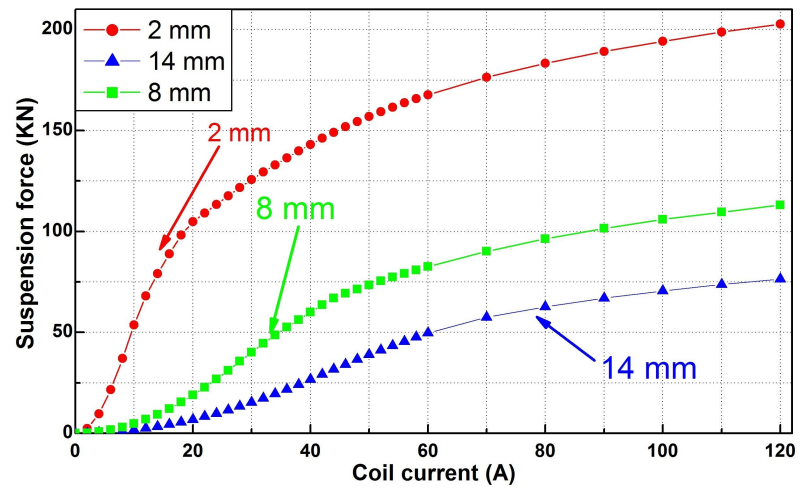

Figure 5: The relationship between the suspension force and input current for three different air gaps

The comparisons of suspension and guidance forces calculated using the two analytic methods and by the FEM

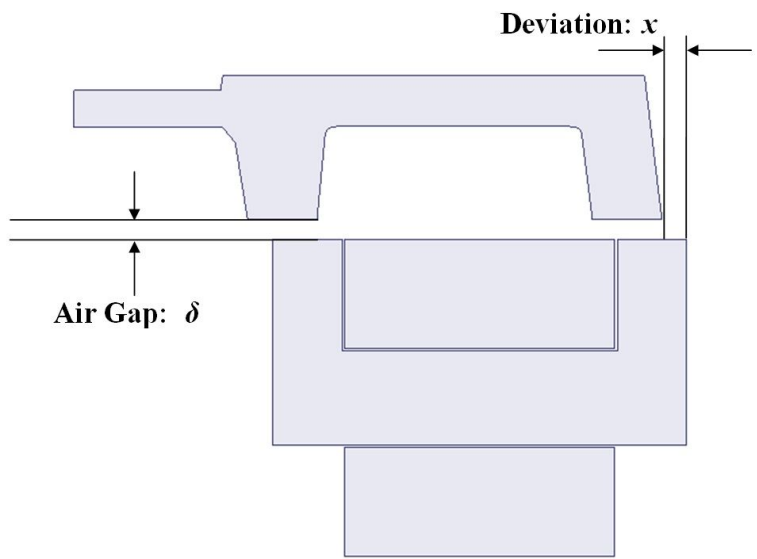

Figure 6: The electromagnetic magnet after lateral offset (with air gap $\delta$ and deviation $x$ )

are presented in Figures 7 and 8 . The air gap between the steel track and the yoke is set to $8 \mathrm{~mm}$ and the input current of the winding is $30 \mathrm{~A}$. It can be seen that the value of the guidance force calculated from (5) is closer to the FEM simulation results, whereas for the suspension force more deviation is observed. Equation (6) provides more accurate results than (5) when calculating both the suspension and guidance forces. Equation (6) with a correction coefficient of 1.076 can therefore be used as a computationally cheap tool instead of directly applying the time-consuming FEM modeling during the design optimization process.

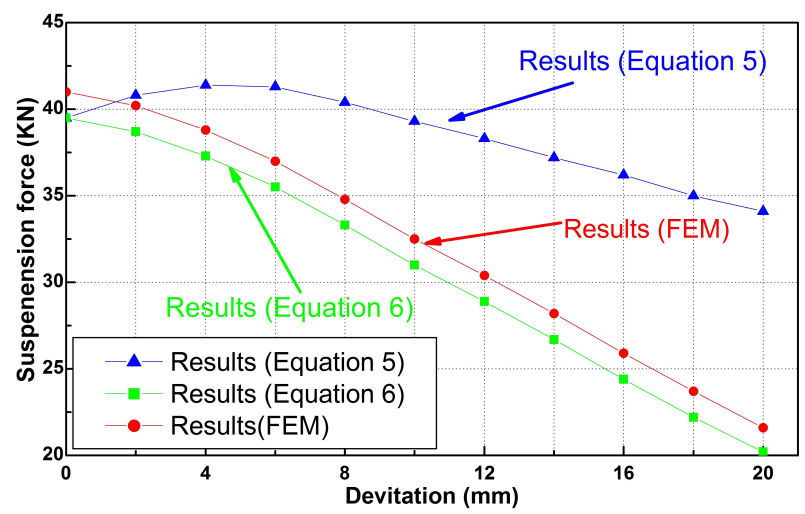

Figure 7: A comparison of the suspension force calculated by two analytic methods and FEM modeling with different values of deviation

The tendency of the suspension guidance force variation is demonstrated in Figures 9 and 10 for three different deviations ( $5 \mathrm{~mm}, 10 \mathrm{~mm}$ and $20 \mathrm{~mm}$ ) as a function of the input current. It can be seen that both the guidance force and the suspension force decline with the increasing devi- 


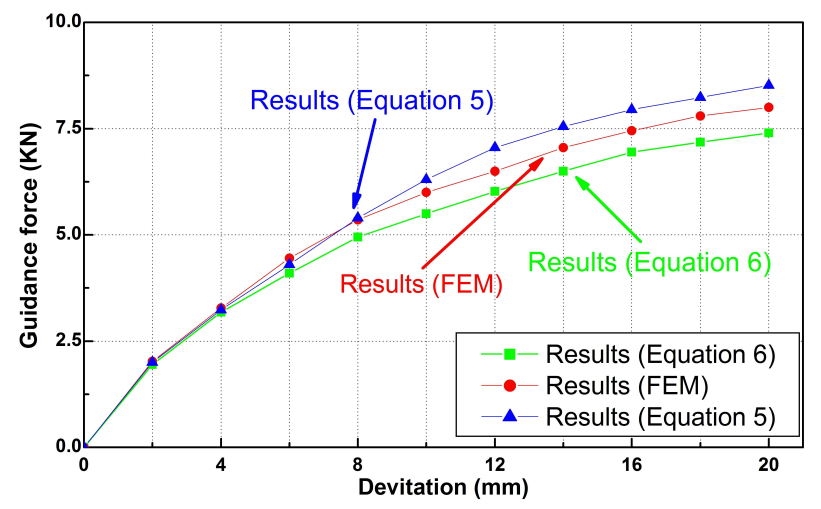

Figure 8: The comparison of suspension force calculated by two analytic methods and FEM modeling with different value of deviation

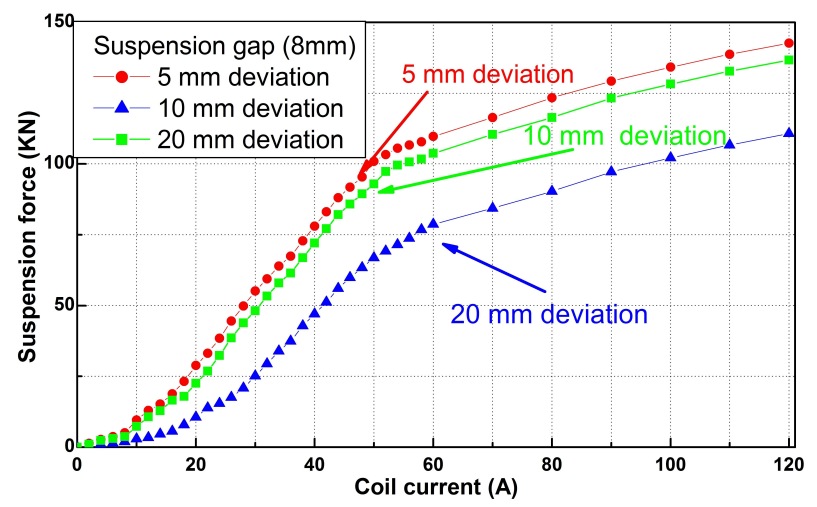

Figure 9: The impact on guidance force of different deviations of the lateral offset as a function of the input current

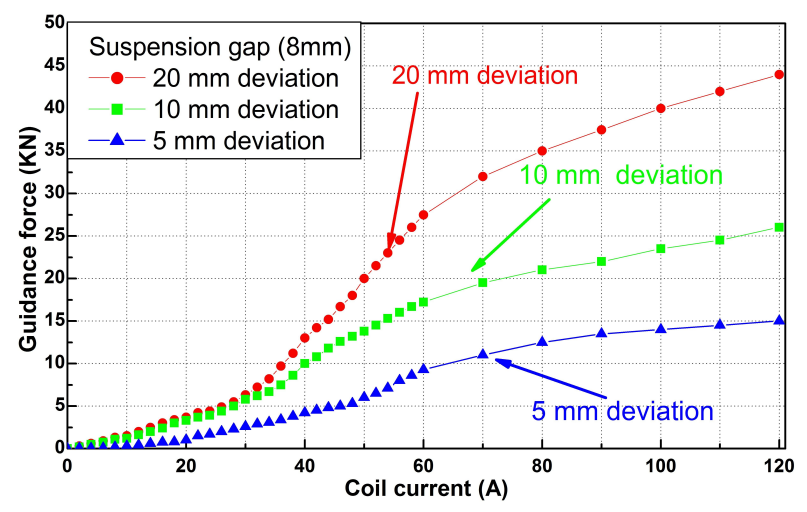

Figure 10: The impact on suspension force of different deviations of the lateral offset as a function of the input current

ation when the air gap is fixed; with the increase of deviation the decreasing rate of the suspension force is gradually enhanced, as shown in Figure 9. Conversely, with the increasing deviation the increasing rate of guidance force gradually reduces in Figure 10.

\section{Permanent-electro-magnetic suspension magnet}

In order to reduce the power loss in the suspension system and increase the force, the concept of a hybrid levitation system - with both permanent magnets and an electromagnet (PEMS) -was proposed in [9-13]. In this paper, three new designs have been pursued which consider protection against mechanical contact. Simulation results of the first prototype show that the electromagnet with embedded permanent magnets can increase the available suspension force by $1.07 \mathrm{kN}$ to $41.24 \mathrm{kN}$, compared to a conventional design. By conducting a series of numerical tests, a location of the magnets as shown in Figure 11 was accomplished (Plan A).

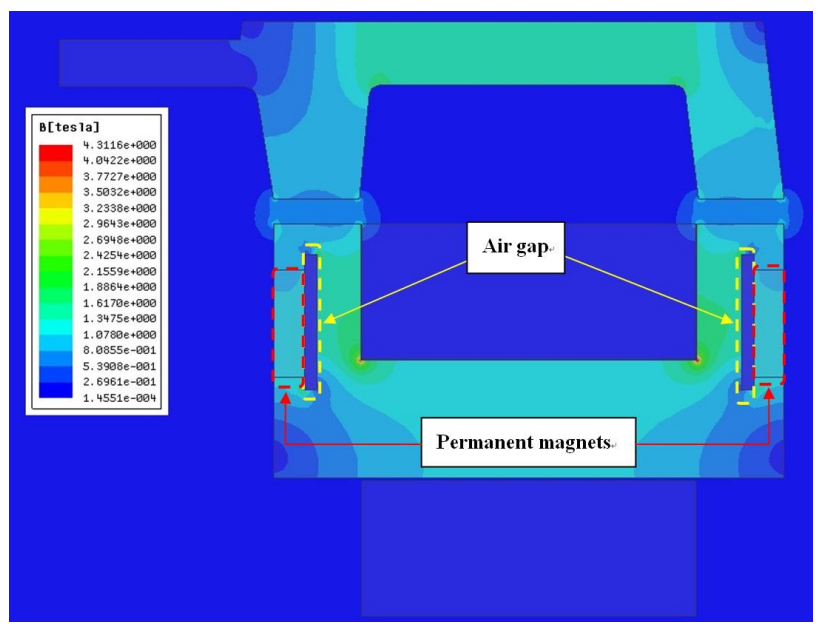

Figure 11: Flux density distribution in Plan A of the PEMS system

Another scheme of the permanent magnets layout is shown in Figure 13. Here (Plan B) the permanent magnet is positioned at the center of the main magnetic circuit and the same power is supplied to the coils as in Plan A. The suspension force is increased slightly by $0.08 \mathrm{kN}$ to $41.32 \mathrm{kN}$.

In order to maintain the balance of electromagnetic forces provided by both the permanent magnets and the electromagnet, a prototype including double pancake coils and a central permanent magnet was considered (Plan C). Because a larger permanent magnet was used the performance is dramatically better than for the former two cases, with the suspension force reaching $51.20 \mathrm{kN}$, as shown in Figure 13. 


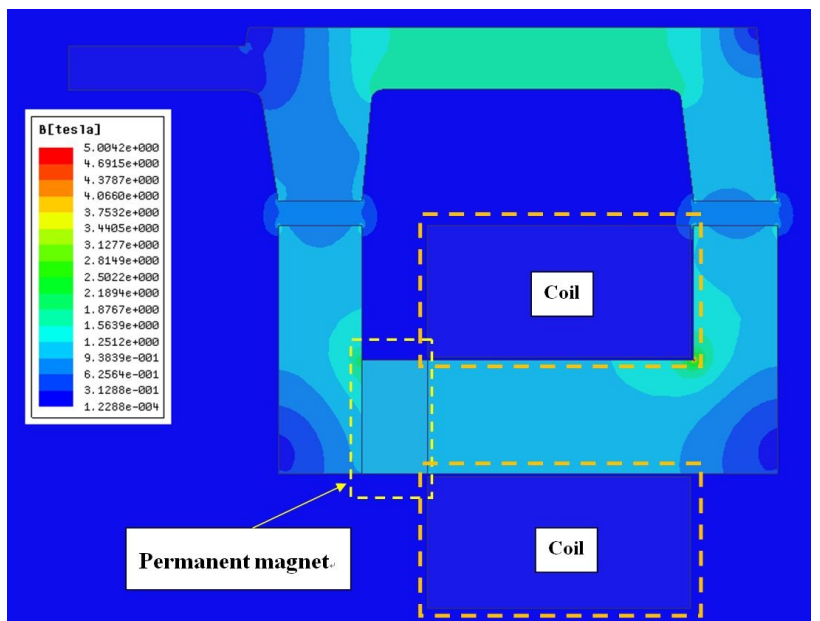

Figure 12: Flux density distribution in Plan B of the PEMS system

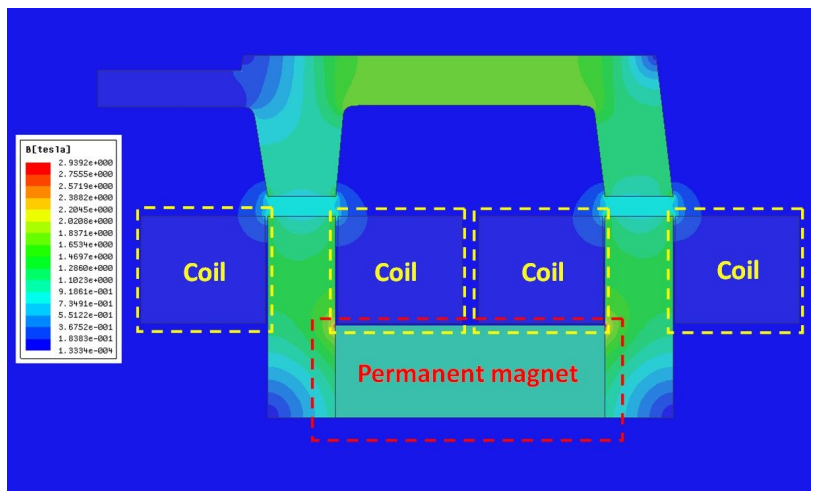

Figure 13: Flux density distribution in Plan C of the PEMS system

\section{Optimal design of the hybrid suspension magnet}

Based on the comparison of the results for the three PM layouts, the third design (Plan C) performs better than the other two, thus it was chosen for further optimization. Five main variables defining the permanent magnet and the iron yoke were selected for optimization as shown in Table ??, while the remaining parameters were fixed as already given in Table 2 . Our previously developed kriging optimizer [14] was used to address this four-variable optimization task.

The kriging optimizer required 492 calls of the FEM simulation software before converging at the optimum solution $\left(H_{p}=35.2 \mathrm{~mm}, W_{p}=138.5 \mathrm{~mm}, h=43.9 \mathrm{~mm}\right.$, $W_{r}=29.3 \mathrm{~mm}, W_{s}=29.8 \mathrm{~mm}$ ), which provides the suspension force of $53.72 \mathrm{kN}$, hence a noticeable improvement. To fully validate the hybrid suspension design under high speed scenario a transient model will be developed, as the next step, to capture the end effects.
Table 2: Test Settingsfor Optimization

\begin{tabular}{ccc}
\hline System parameters & Symbol & $\begin{array}{c}\text { Test range } \\
{[\mathrm{mm}]}\end{array}$ \\
\hline Vertical height of the permanent magnet & $H_{p}$ & {$[2438]$} \\
Horizontal width of the permanent magnet & $W_{p}$ & {$[62167]$} \\
Magnetic yoke height of electromagnet & $h$ & {$[3545]$} \\
Magnetic pole width of steel rail & $W_{r}$ & {$[2030]$} \\
Magnetic pole width of electromagnet & $W_{s}$ & {$[2030]$} \\
\hline
\end{tabular}

\section{Conclusions}

The performance of a suspension system of maglev trains has been investigated from the point of view of the dependence of the force on the size of the air gap and value of the current. Improvements to the conventional system were accomplished by implementing a concept of a hybrid arrangement of electromagnets and permanent magnets. It is argued that the hybrid system improves the characteristics by increasing the available force while reducing associated losses.

\section{References}

[1] Tzeng Y.K. Tsih C.W., A novel compensating approach for selfsensing maglev system with controlled-PM electromagnets, IEEE Transactions on Magnetics, 1995, 31, 4208-4210.

[2] Xu Z.G., Jin N.Q., Shi L.M., et al., Maglev system with hybridexcited magnets and an air-gap length control, Proceedings of Maglev' 2004, China, 2004, 1019-1023.

[3] Toshio K., Hitoshi Y., Katsuji S., Energy-saving coil excitation of electromagnetic levitation system using divided iron core type hybrid magnet, IEEE Transactions on Industry Applications, 2007, 127, 935-941.

[4] Cho H.W., Han H.S., Lee J.M., et al., Design considerations of EMPM hybrid levitation and propulsion device for magnetically levitated vehicle, IEEE Transactions on Magnetics, 2009, 45, 4632 4635.

[5] Shaoke L., Honghan N., Kuikui Z., Analysis on guidance ability for mid and low speed maglev train, Electric Drive for Locomotives, 2007, 32, 36-38.

[6] Liu S., An B., Liu S., Guo Z., Characteristic research of electromagnetic force for mixing suspension electromagnet used in low-speed maglev train, Electric Power Applications IET, 2015, 9, 223-228.

[7] Han H., Moon S., Cho H., State-of-the-art multibody dynamic simulations of EMS-type maglev vehicles at KIMM, San Diego, 2008, 12, 15-19.

[8] Arrigo A.D., Rufer A., Design of integrated electromagnetic levitation and guidance system for Swiss Metro, International Journal of Immunogenetics, 1999, 32, 343-34.

[9] Han W.T., Sun J.J., Liu X.K., Guo H.X., Wang J.S., A novel hybrid suspension electromagnet for middle-low speed maglev train, 
Journal of Magnetics, 2017, 22, 463-471.

[10] Li Y.G., Cheng H., Long Z.Q., Stability analysis and controller design of hybrid EMS maglev system, Proceedings of 8th ISMST, Dresden, 2005, 126-130.

[11] Toshio K., Hitoshi Y., Katsuji S., Energy-saving coil excitation of electromagnetic levitation system using divided iron core type hybrid magnet, IEEE Transactions on Industry Applications, 2007, 127, 935-941.

[12] Cho H.W., Han H.S., Lee J.M., et al., Design considerations of EMPM hybrid levitation and propulsion device for magnetically levitated vehicle, IEEE Transactions on Magnetics, 2009, 45, 4632-4635.
[13] Kehrer K., Mc Kenna W., Shumaker W., Maglev design for PM levitation Electrodynamic Suspension (EDS) system, Proceedings of Maglev'2004, Switzerland, 2002, 120-126.

[14] Xiao S., Rotaru M., Sykulski J.K., Adaptive weighted expected improvement with rewards approach in kriging assisted electromagnetic design, IEEE Transactions on Magnetics, 2013, 49, 2057-2060. 